\title{
Apolipoprotein A-IV in the Follicle-Associated Epithelium: A Further Piece in the Puzzle
}

\author{
Evelyn Orsó • Gerd Schmitz
}

Published online: 19 September 2014

(c) Springer Science+Business Media New York 2014

Apolipoprotein A-IV (apoA-IV) is a 46-kDa exchangeable plasma apolipoprotein, secreted primarily by the intestinal mucosa and to a lesser extent by hepatocytes in humans and in most animals. Since its first description in 1974, a variety of functions has been proposed for apoA-IV, including involvement in the assembly, secretion, and metabolism of lipids, in the regulation of food intake as a satiety factor and modulator of gastric acid secretion, and in the protection against inflammation and atherosclerosis due to its antioxidant properties [1 and references therein]. ApoA-IV is synthesized in villous enterocytes (VE) and assembled to pre-chylomicron transport vesicles in the Golgi complex. The latter contributes to the maturation of pre-chylomicron particles along their vectorial transport to the basolateral surface, prior to secretion into the pericellular spaces adjacent to lymphatic fenestrae [2]. Despite considerable effort, the precise cellular function of apoAIV in enterocytes has not yet been elucidated.

The follicle-associated epithelium (FAE) is a poorly characterized region of the intestinal mucosa that overlies mucosa-associated lymphoid tissue (e.g., Peyer's patches). The FAE is clearly different from VE, since there is a high density of $M$ (microfold) cells, specialized for antigen sampling and transcytosis of IgA, parallel with the lack goblet cells and subepithelial myofibroblasts [3 and references therein]. It is unclear whether FAE is comprised in part of predetermined $\mathrm{M}$ cell precursors, since the mRNA profile of FAE differs from the transcriptome of $\mathrm{M}$ cells or

E. Orsó $(\bowtie) \cdot$ G. Schmitz

Institute for Laboratory Medicine and Transfusion Medicine, University Hospital Regensburg, Franz-Josef-Strauss-Allee 11, 93053 Regensburg, Germany

e-mail: evelyn.orso@klinik.uni-regensburg.de transcriptome of VE cells [3]. The FAE is considered as a major component of mucosal immune response.

In this issue of Digestive Diseases and Sciences, Tokuhara et al. [4] provide evidence that apoA-IV mRNA and protein are constitutively expressed in cells of the FAE, but absent in $\mathrm{M}$ cells in the mouse small intestine. Moreover, mice strongly express apoA-IV protein in the jejunal VE before weaning, but surprisingly not in chowfed young (3-week-old) or adult (7-week-old) mice [4]. In addition to protein expression in the FAE, apoA-IV mRNA is expressed in jejunal VE during development before weaning, which continuously declines after weaning [4]. In adult mice, the mRNA expression of apoA-IV is largely restricted to the FAE and to the tips of jejunal VE [4].

A monoclonal antibody generated by the authors against mouse FAE recognized apoA-IV with high specificity [4], serving as a valuable and unique tool for apoA-IV protein localization, which had not been reported previously, due to lack of antibody specificity [1, 4 and references therein].

Considering the recent findings of Tokuhara et al. [4], the amount of apoA-IV expression in adult mouse VE is most likely very low in the basal or fasting state, with rapid induction following lipid ingestion, consistent with apoAIV expression in the VE of suckling mice [4], and with the extensive regulation of apoA-IV by nutritional and hormonal factors such as the fat in breast milk [1 and references therein].

Constitutive, age-independent expression of apoA-IV mRNA and protein in the mouse FAE is an important novel finding of Tokuhara et al. [4], adding a further element to the characterization of apoA-IV and to adjacent non-M cells in the FAE. The expression of apoA-IV is not mandatory for the organogenesis of Peyer's patches, as apoAIV-deficient mice (global knockout) have morphologically intact Peyer's patches [4] and develop no major 


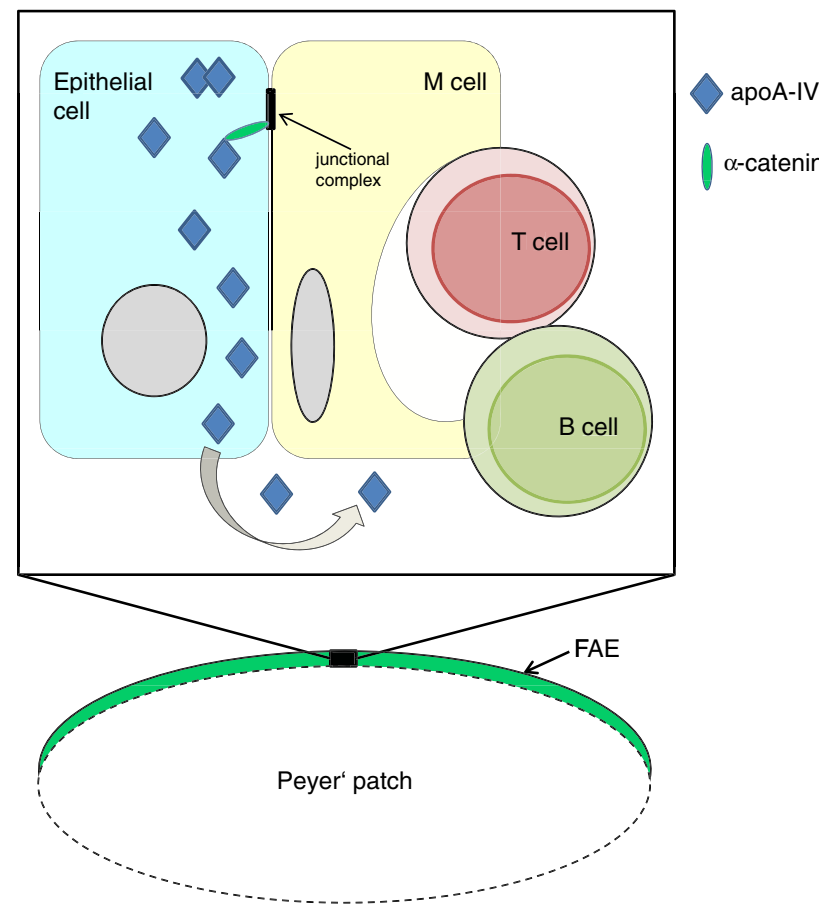

Fig. 1 Scheme of the cellular complexity and the potential role of apoA-IV in follicle-associated epithelium

immunodeficiency [5]. Additional studies of how apoA-IV might influence the biogenesis or differentiation of $\mathrm{M}$ cells in the FAE, or the "fine tuning" of Peyer's patches, for instance, in cell- or tissue-specific apoA-IV deficiency (flox) or knockdown approaches, might be of interest.

One important question that needs to be addressed is the functional significance of apoA-IV expression in the FAE, especially in the apoA-IV-positive cells surrounding $M$ cells. A prior analysis of the molecular interactions with apoA-IV [6] might help further clarify this issue: apoA-IV may contribute to the stability of junctional integrity in FAE through its interaction with $\alpha$-catenin [6] and thus promote or inhibit the differentiation of adjacent $\mathrm{M}$ cells, influence the permeability of FAE for luminal commensal or pathogenic bacteria to induce specific immune responses in Peyer's patches, or modulate the extension of dendrites of specific antigen-presenting cells in the dome (Fig. 1). ApoA-IV may also function in the VE as intracellular adaptor or chaperone through its interaction with apolipoprotein B-48 (apoB), which contributes to the proper assembly of chylomicrons. In this respect, apoA-IV might work as a cytoplasmic adaptor for metabolic and likely also for immunologic learning in the intestine, as previously postulated by Schmitz and Langmann [7]. The cell cycle of FAE or VE may be influenced by apoA-IV through its interaction with cyclin C [6].

A further possibility is that apoA-IV is actively secreted from FAE, acting on the neighboring immune cells or FAE as a paracrine or autocrine modulator, respectively (Fig. 1). In this situation, the factors contributing to apoA-IV secretion from FAE have yet to be identified, with Toll-like receptors (TLRs) likely candidates. Expression of TLR2, TLR5, and TLR9 mRNA and protein was reported recently at apical and basolateral sites of the FAE in mice [8]. Endogenous apolipoprotein A-I (apoA-I), a close molecular relative of apoAIV, is actively secreted from human macrophages in response to Toll-like receptor-4 signaling, stabilizing lipid rafts and the ATP-binding cassette transporter A1 (ABCA1), one of the major mediators of cellular lipid efflux [9]. Since apoA-IV was evolutionarily derived by duplication of the apoA-I gene, with both located in a common gene cluster on human chromosome 11, these apolipoproteins share many properties [10 and references therein]. Thus, apoA-IV may also be secreted from FAE, modulating diverse cellular functions from the extracellular site through receptor interactions and signaling mechanisms.

In summary, Tokuhara et al. [4] have advanced the field by identifying constitutive apoA-IV expression in FAE and by demonstrating age-dependent apoA-IV expression in VE, thus opening up new opportunities for understanding apoA-IV actions in the intestinal mucosa.

\section{References}

1. Duka A, Fotakis P, Georgiadou D, et al. ApoA-IV promotes the biogenesis of apoA-IV-containing HDL particles with the participation of ABCA1 and LCAT. J Lipid Res. 2013;54:107-115.

2. Abumrad NA, Davidson NO. Role of the gut in lipid homeostasis. Physiol Rev. 2012;92:1061-1085.

3. Finke D. Introduction of intestinal lymphoid tissue formation by intrinsic and extrinsic signals. Semin Immunopathol. 2009;31: 151-169.

4. Tokuhara D, Nochi T, Matsumura A, et al. Specific expression of apolipoprotein A-IV in the follicle-associated epithelium of the small intestine. Dig Dis Sci. (Epub ahead of print). doi:10.1007/ s10620-014-3203-6.

5. Weinstock PH, Bisgaier CL, Hayek T, et al. Decreased HDL cholesterol levels but normal lipid absorption, growth, and feeding behavior in apolipoprotein A-IV knockout mice. J Lipid Res. 1997;38:1782-1794.

6. Orsó E, Moehle C, Boettcher A, et al. The satiety factor apolipoprotein A-IV modulates intestinal epithelial permeability through its interaction with \& $\alpha$-catenin: implications for inflammatory bowel diseases. Horm Metab Res. 2007;39:601-611.

7. Schmitz G, Langmann T. Metabolic learning in the intestine: adaptation to nutrition and luminal factors. Horm Metab Res. 2006;38:452-454.

8. Abreu MT. Toll-like receptor signaling in the intestinal epithelium: how bacterial recognition shapes intestinal function. Nat Rev Immunol. 2010;10:131-143.

9. Mogilenko DA, Orlov SV, Trulioff AS, et al. Endogenous apolipoprotein A-I stabilizes ATB-binding cassette transporter A1 and modulates Toll-like receptor 4 signaling in human macrophages. FASEB J. 2012;26:2019-2030.

10. Simon T, Cook VR, Rao A, Weinberg RB. Impact of murine intestinal apolipoprotein A-IV expression on regional lipid absorption, gene expression, and growth. J Lipid Res. 2011;52:1984-1994. 